\title{
Does Accounting for Gene-Environment Interactions Help Uncover Association between Rare Variants and Complex Diseases?
}

\author{
Rémi Kazma Niall J. Cardin John S. Witte \\ Department of Epidemiology and Biostatistics and Institute for Human Genetics, University of California, \\ San Francisco, Calif., USA
}

\section{Key Words}

Rare variant - Gene-environment interaction · Association •

Case-control design · Logistic regression · Simulation · Power

\begin{abstract}
Objective: To determine whether accounting for gene-environment $(\mathrm{G} \times \mathrm{E})$ interactions improves the power to detect associations between rare variants and a disease, we have extended three statistical methods and compared their power under various simulated disease models. Methods: To test for association of a group of rare variants with a disease, Min-P uses the lowest $p$ value within the group of variants, CAST (Cohort Allelic Sums Test) uses an indicator variable to quantify the rare alleles within the group of variants, and SKAT (Sequence Kernel Association Test) uses a logistic regression based on kernel machine. For each method, we incorporate a term for the GXE interaction and test for association and interaction jointly. Results: When testing for disease association with a set of rare variants, accounting for $\mathrm{G} \times \mathrm{E}$ interactions can improve power in specific situations (pure interaction or high proportion of causal variants interacting with the environment). However, the power of this approach can decrease, in particular in the presence of main genetic or environmental effects. Among the methods compared, the optimized and weighted SKAT performed best,
\end{abstract}

whether to test for genetic association or to test it jointly with GXE interactions. Conclusion: This approach can be used in specific situations but is not appropriate for a primary analysis.

Copyright $\odot 2013$ S. Karger AG, Basel

\section{Introduction}

Rare genetic variant association and gene-environment $(\mathrm{G} \times \mathrm{E})$ interaction are often quoted to explain the missing heritability observed in association studies of complex diseases with common genetic variants [1-4]. In contrast with the common disease - common variant hypothesis, where the burden of disease causality is thought to lie in the high relative frequency of the alleles associated with the disease, the common disease - rare variants hypothesis relies on the high number and expected high penetrance of these variants to explain part of the disease heritability [5-7]. There is evidence to suggest that both models are relevant to at least some common human diseases [8], but until recently, information on rare variants has been limited by the high cost of sequencing. With the advent of next-generation sequencing and whole-exome arrays, large-scale genotyping of rare variants with exome arrays and reasonably large sequencing projects are becoming affordable. But the pow-

\section{KARGER}

E-Mail karger@karger.com

www.karger.com/hhe
(C) 2013 S. Karger AG, Basel

0001-5652/12/0744-0205\$38.00/0
John S. Witte, $\mathrm{PhD}$

MC 3110, UCSF, Helen Diller Family Cancer Research Building

1450 3rd Street, PO Box 589001

San Francisco, CA 94158-9001 (USA)

E-Mail WitteJ@ humgen.ucsf.edu 
er of traditional statistical methods is expected to be low due to the sparsity of rare variant data.

To improve the power for detecting association with rare variants, many methods have been proposed [9-21], as yet, none of them directly account for potential $\mathrm{G} \times \mathrm{E}$ interactions. When evaluating common variants, testing for genetic association and $\mathrm{G} \times \mathrm{E}$ interaction jointly can improve the power to detect association in specific situations, but this strategy is also subject to a power decrease in the absence of $\mathrm{G} \times \mathrm{E}$ interaction $[22,23]$.

To determine whether accounting for $\mathrm{G} \times \mathrm{E}$ interactions when testing for disease association with rare genetic variants increases power, we have extended three statistical methods used in rare variant association testing, by incorporating an interaction term and testing jointly for genetic association and $\mathrm{G} \times \mathrm{E}$ interaction. In this paper, we present these methods and compare their statistical properties under several simulated disease models with and without $\mathrm{G} \times \mathrm{E}$ interactions involving rare genetic variants.

\section{Methods}

\section{Notation}

Consider a genetic region $\mathrm{G}$, a dichotomous environmental exposure $\mathrm{E}$ and a dichotomous disease D. Region $\mathrm{G}$ can be defined by a gene coding for a protein, by a region corresponding to noncoding RNA, by a region previously found to be linked or associated to $\mathrm{D}$, or by any other arbitrary segment of the genome. The genetic variability of $G$ in the general population is such that $G$ harbors $m$ variants. Exposure E has population prevalence $f_{E}$ and is assumed to be independent of the $m$ variants in $G$ in the population. Disease $\mathrm{D}$ is associated with a subset $p$ of the $m$ variants in $\mathrm{G}$ (assuming an additive model of effect) and to E, following the logistic model:

$$
\operatorname{logit} P\left(y_{i}=1\right)=\beta_{0}+\sum_{k=1}^{p} \beta_{G k} g_{k i}+\beta_{E} e_{i}+\sum_{k=1}^{p} \beta_{I k} g_{k i} e_{i}
$$

where $y_{i}$ is the disease phenotype of individual $i ; \beta_{0}$ is $\log (\mathrm{B} /(1-$ $\mathrm{B})$ ), $\mathrm{B}$ being the baseline disease risk for an individual unexposed and non-carrier of any rare allele; $g_{k i}$ is the genotype count (i.e. 0,1 , or 2 minor alleles) for individual $i$ at the $k$-th of the $p$ variants in $\mathrm{G}$ associated with $\mathrm{D}$ (variant $k$ ); $\beta_{G k}$ is the coefficient of association between variant $k$ and $\mathrm{D}$ (in the absence of $\mathrm{E}$ ); $e_{i}$ is the exposure status of individual $i$ to $\mathrm{E} ; \beta_{E}$ is the coefficient of association between $\mathrm{E}$ and $\mathrm{D}$ (in the absence of minor alleles at any of the $p$ variants), and $\beta_{I k}$ is the coefficient of $\mathrm{G} \times \mathrm{E}$ interaction of variant $k$ and $\mathrm{E}$.

To test for the association between $\mathrm{G}$ and $\mathrm{D}$, while accounting for potential $\mathrm{G} \times \mathrm{E}$ interactions, we have extended three methods: Min-P, the Cohort Allelic Sums Test (CAST), and the SNP-set (Sequence) Kernel Association Test (SKAT), incorporating a G×E interaction term and testing jointly for genetic association and $\mathrm{G} \times \mathrm{E}$ interaction.

\section{Min-P}

A simple approach is to analyze the association between each of the $m$ variants and $\mathrm{D}$ and to select the $\mathrm{p}$ value of the most significantly associated rare variant (i.e. Min-P) as a test statistic for the association with the region. To ensure proper control of the type 1 error, a permutation procedure of the case-control labels estimates the empirical distribution of Min-P and the $\mathrm{p}$ value is calculated as the fraction of Min-P values in permutations that are at least as extreme as the observed Min-P [24]. The association test used can be a likelihood ratio test based on a generalized linear model, a Cochran-Armitage trend test, or a Fisher exact test. However, because of potential data sparsity, here we used the Fisher exact test [25].

To test for genetic association only, the Fisher exact test is calculated for the contingency table crossing D and each of the $m$ variants. This test will be noted Min-P-g. To test jointly for genetic association and $\mathrm{G} \times \mathrm{E}$ interaction, we propose to stratify the data on $\mathrm{E}$ and do two Fisher exact tests on contingency tables crossing $\mathrm{D}$ and each of the $m$ variants in exposed and unexposed individuals. To combine the two independent tests, we used the Fisher product method. This test will be noted Min-P-ge.

Because of the computational burden of the Fisher exact test, we used an adaptive permutation procedure (up to 1,000 permutations) to estimate the empirical Min-P distributions. Thereby, the low resolution for small $\mathrm{p}$ values $(<0.01)$ does not allow estimating the power at a genome-wide level (requiring at least resolution up to $10^{-5}$ ).

\section{Cohort Allelic Sums Test}

CAST was the first burden testing method introduced to aggregate rare variants when testing for disease association [16]. An indicator variable $\left(S_{i}\right)$ is generated for each individual $i$, such that $S_{i}$ equals 0 in the absence of minor alleles or 1 in the presence of at least one minor allele among all variants below a predefined threshold $\mathrm{T}$ for the minor allele frequency (MAF) in the sample (binary coding). To accommodate potential additive effects of rare alleles, $S_{i}$ can also be the count of minor alleles below $\mathrm{T}$ (additive coding) [17].

To test for genetic association only, we included $S_{i}$ as an independent variable in a logistic regression on the disease statuses $y_{i}$, and a likelihood ratio was used to test the null hypothesis $H_{0}$ : $\beta_{G S}=0$. This test will be noted CAST-g.

$$
\operatorname{logit} P\left(y_{i}=1\right)=\beta_{0}+\beta_{G S} S_{i}
$$

To test jointly for genetic association and $\mathrm{G} \times \mathrm{E}$ interaction, we propose to incorporate a $\mathrm{G} \times \mathrm{E}$ interaction term $\left(\beta_{I S}\right)$ and used a likelihood ratio to test $H_{0}: \beta_{G S}=\beta_{I S}=0$. This test will be noted CAST-ge.

$$
\operatorname{logit} P\left(y_{i}=1\right)=\beta_{0}+\beta_{G S} S_{i}+\beta_{E} e_{i}+\beta_{I S} S_{i} e_{i}
$$

The power of CAST relies partly on choosing the right threshold $\mathrm{T}$ and coding (binary or additive) for calculating $S_{i}$. A balanced case-control sampling increases the sample MAF of associated variants compared to their population MAF. Therefore, in our comparison, we evaluated the impact of these choices by comparing the use of two values of $\mathrm{T}(0.05$ and 0.01$)$ and both codings for calculating $S_{i}$ (binary and additive).

\section{Sequence Kernel Association Test}

To accommodate the analysis of a set of rare variants, while adjusting for covariates, SKAT uses a logistic kernel machine 
framework aggregating individual score test statistics of variants and provides a global $p$ value for the set of variants tested $[20,26]$.

Assuming that each $\beta_{G m}$ (i.e. the association coefficients of all variants $m$ in the set) independently follows an arbitrary distribution with mean 0 and variance $\omega_{m}{ }^{2} \psi$, where $\omega_{m}$ is a weight for each of the $m$ variants, testing $H_{0}: \beta_{G m}=0$ is equivalent to testing $\psi=0$ (i.e. a variance-component test score done using the corresponding mixed model). For a case-control design with $n$ individuals sampled and $m$ variants genotyped, $\mathbf{G}$ is the $n \times m$ matrix of genotypes, $\mathbf{W}$ is the $m \times m$ diagonal matrix of weights $\left(\omega_{m}\right)$, and $\mathbf{K}=$ $\mathbf{G W W G}^{\mathrm{T}}$ is the $n \times n$ weighted linear kernel matrix, which defines the genetic similarity between all pairs of individuals. The function that links each element of the matrix $\mathbf{K}$ to $\mathbf{G}$ is the kernel function.

To test for genetic association only, the variance-component

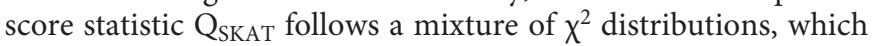
are closely approximated with the Davies method [27].

$$
\mathrm{Q}_{\text {SKAT }} \cong(\mathbf{y}-\bar{y})^{\mathrm{T}} \mathbf{K}(\mathbf{y}-\bar{y})
$$

where $\bar{y}$ is the predicted mean of the vector of disease status values (y) under $H_{0}$, obtained by regressing y on the adjustment covariates only.

Two important decisions have to be considered when using SKAT for analyzing rare variants: the choice of a weighting scheme and the choice of a kernel. Using an identity matrix of size $m$ for the weight matrix $\mathbf{W}$ gives an equal weight to all variants. When focusing on rare variant analysis, one might want to put more weight on rarer than on more common variants. This has been shown to improve power, in particular if the lower the MAF, the higher the variant's effect. For example, Madsen and Browning [15] proposed to use the inverse of the MAF variance to give more weight to rarer variants. In the SKAT method, Wu et al. [20] proposed to weight variants using a Beta(MAF, $a_{1}, a_{2}$ ) distribution with parameters $a_{1}=1$ and $a_{2}=25$, allowing higher weights for rarer variants while still giving some weight to variants with a MAF between 0.01 and 0.05 .

Several kernels have been suggested by the authors of this method. In fact, most of the tests and variant groupings proposed in the literature for analyzing rare variants can be rewritten as particular cases of SKAT, by using appropriate weights and kernels. However, it is difficult to know a priori which kernel to use for a given set of data and using the weighted linear kernel may not always be the best approach. In particular, if a large proportion of variants is associated with the disease with the same direction of effect, simple burden tests are often more powerful. To overcome this issue, Lee et al. [28] have recently proposed to optimize the kernel by computing the best linear combination of SKAT using a weighted linear kernel (noted SKAT hereafter) and the equivalent of the score test statistic of the weighted additive burden test (noted Burden). By allowing the $\beta_{G m}$ to follow a multivariate distribution with exchangeable relation structure, using the correlation matrix $\mathbf{R}_{\rho}=$ $(1-\rho) \mathrm{I}+\rho \mathbf{1} \mathbf{1}^{\mathrm{T}}$, the SKAT test statistic becomes a function of $\rho$ :

$$
\mathrm{Q}_{\rho} \cong(\mathbf{y}-\bar{y})^{\mathrm{T}} \mathbf{K}_{\rho}(\mathbf{y}-\bar{y})
$$

where $\mathbf{K}_{\rho}=\mathbf{G} \mathbf{W} \mathbf{R}_{\rho} \mathbf{W} \mathbf{G}^{\mathrm{T}}$. When $\rho=0, \mathbf{K}_{\rho}$ is the weighted linear kernel and $\mathrm{Q}_{\rho}$ is equal to $\mathrm{Q}_{\mathrm{SKAT}}$ in equation 4 . When $\rho=1, \mathbf{K}_{\rho}$ is the weighted burden test and $\mathrm{Q}_{\rho}$ is equal to:

$$
\mathrm{Q}_{\text {BURDEN }} \cong(\mathbf{y}-\bar{y})^{\mathrm{T}} \mathbf{G W} \mathbf{1 1} \mathbf{1}^{\mathrm{T}} \mathbf{W G}^{\mathrm{T}}(\mathbf{y}-\bar{y})
$$

which is equivalent to the square of the score test statistic of the weighted burden test. Thus, $\mathrm{Q}_{\rho}$ is a linear combination of SKAT and the Burden test, i.e. $\mathrm{Q}_{\rho}=(1-\rho) \mathrm{Q}_{\mathrm{SKAT}}+\rho \mathrm{Q}_{\mathrm{BURDEN}}$. Optimization of this linear combination is achieved by maximizing the test statistic over a range of correlation structures between the variants' effects (i.e. the combination with a minimum p value). However, the empirical distribution of this test (noted SKAT-O) is derived analytically, thus avoiding the computational burden of permutation testing [28].

To test jointly for genetic association and $\mathrm{G} \times \mathrm{E}$ interaction, we propose to replace the $n \times m$ matrix $\mathbf{G}$ with an $n \times 2 m$ matrix $\mathbf{J}$, where half of the matrix contains the genotypes $g_{m i}$ and the other half contains the $\mathrm{G} \times \mathrm{E}$ interaction terms $g_{m i} e_{i}$. Replacing $\mathbf{G}$ with $\mathbf{J}$ in equations 4-6 provides variance-component score statistics for the joint test of genetic association and $\mathrm{G} \times \mathrm{E}$ interaction using SKAT, SKAT-O, and the weighted burden test, respectively.

In our comparison, to test for genetic association only or genetic association and $\mathrm{G} \times \mathrm{E}$ interaction jointly, we used the linear kernel-based test (noted SKAT-g and SKAT-ge), the optimized test (noted SKAT-O-g and SKAT-O-ge), and the weighted burden test (noted Burden-g and Burden-ge). For these six tests, we used weights based on a Beta(MAF, $a_{1}=1, a_{2}=25$ ) distribution for the matrix part corresponding to genotypes and on a $\operatorname{Beta}\left(f_{\mathrm{G} \times \mathrm{E}}, a_{1}=1\right.$, $\left.a_{2}=25\right)$ distribution, where $f_{\mathrm{G} \times \mathrm{E}}$ is the relative frequency of the $\mathrm{G} \times \mathrm{E}$ terms $\left(f_{\mathrm{G} \times \mathrm{E}}=\Sigma\left(g_{i} e_{i}\right) / 2 n\right)$, for the matrix part corresponding to $\mathrm{G} \times \mathrm{E}$ interactions.

\section{Simulations}

To compare the performance of the different methods with and without accounting for $\mathrm{G} \times \mathrm{E}$ interactions, we simulated case-control samples under a range of disease models using a set of 10,000 haplotypes simulated over a 4.7-Mb stretch of chromosome 17 (as in [29]). These haplotypes were simulated based on population genetic parameters derived from the Seattle SNPs project using the program SFS_CODE [30]. The distribution of the 61,550 polymorphic sites shows a high proportion of rare variants: $21.3 \%$ were singletons, $10.1 \%$ were doubletons, $34.7 \%$ had a MAF $<0.001$ (not including singletons and doubletons), $24.6 \%$ had a MAF between 0.001 and 0.01 , and $7.6 \%$ had a MAF between 0.01 and 0.05 .

The disease models included two scenarios. The first considers genetic regions of 20,100 , or 400 variants with $10 \%$ of the variants directly associated with $\mathrm{D}$, whereas the second considers genetic regions of 20 variants with 25,50 , or $75 \%$ of the variants directly associated with D. In practice, scenario 1 corresponds to an agnostic approach where all variants are included in the analysis and the proportion of causal variants is expected to be relatively low, whereas scenario 2 corresponds to an analysis performed after pruning to select the potentially most interesting variants using various criteria (e.g. variants that are non-synonymous, deleterious, or not previously referenced). Taking the 10,000 haplotypes in pairs generated 5,000 diploid individuals. Then, non-overlapping bins of 20,100 , and 400 variants with a population MAF between $3 \times 10^{-4}$ and 0.5 defined the genetic regions in scenario 1 , whereas in scenario 2, the 20 variants were selected to have a population MAF between $3 \times 10^{-4}$ and 0.05 . The purpose of excluding common variants (MAF $>0.05$ ) in scenario 2 was again to mimic an analysis focusing on pre-selected rare variants. In both scenarios, the risk variants were selected at random without replacement among variants with a population MAF in the range between $3 \times$ $10^{-4}$ and 0.05 . Although we included variants with a MAF between 
Table 1. MAF distribution of variants in the simulated regions

\begin{tabular}{|c|c|c|c|c|c|c|c|c|c|}
\hline \multirow[t]{2}{*}{ Scenario } & \multicolumn{5}{|c|}{ MAF of all variants $(m)$} & \multicolumn{4}{|c|}{ MAF of causal variants $(p)$} \\
\hline & $m$ & $<0.001$ & $0.001-0.01$ & $0.01-0.05$ & $>0.05$ & $p$ & $<0.001$ & $0.001-0.01$ & $0.01-0.05$ \\
\hline \multirow{3}{*}{1} & & $7[0-16]$ & $7[1-16]$ & $2[0-11]$ & $3[0-13]$ & & $1[0-2]$ & $1[0-2]$ & $0[0-2]$ \\
\hline & 100 & $35.35(5.57)$ & $35.92(5.88)$ & $11.05(4.89)$ & $17.68(6.48)$ & 10 & $3.49(1.52)$ & $5.04(1.64)$ & $1.47(1.22)$ \\
\hline & & $141[104-183]$ & $143[102-184]$ & $42[18-84]$ & $73[17-118]$ & & $14[7-26]$ & $20[13-28]$ & $6[0-14]$ \\
\hline \multirow[t]{3}{*}{2} & 20 & $8.57(2.33)$ & $8.71(2.34)$ & $2.71(1.76)$ & - & 5 & $1.84(1.03)$ & $2.49(1.08)$ & $0.67(0.77)$ \\
\hline & & 9 [3-17] & $9[2-17]$ & $2[0-12]$ & & & $2[0-5]$ & $2[0-5]$ & $1[0-4]$ \\
\hline & & & & & & 10 & $3.72(1.54)$ & $4.92(1.59)$ & $1.36(1.12)$ \\
\hline
\end{tabular}

Values are mean numbers of variants (with standard deviations in parentheses) in the first row and median numbers of variants [with min-max in brackets] in the second row.

0.01 and 0.05 , their odds of being selected first are almost 8 times smaller than the odds of variants $<0.01$. The comparison of the population MAF distribution between causal variants and all variants illustrates the overabundant selection of rare variants just by random sampling (table 1). In scenario 1 , on average $85 \%$ of the selected causal variants have a MAF $<0.01$, of which $42 \%$ have a $\mathrm{MAF}<0.001$. Similar figures are obtained in scenario 2.

For each disease model, the sampling of 1,000 cases and 1,000 controls was done using the additive logistic model in equation 1. The baseline risk of $\mathrm{D}$ was 0.01 and sampling of exposure status was done for each genetic region, setting the prevalence of $\mathrm{E}\left(f_{E}\right)$ equal to 0.3 and the odds ratio of $\mathrm{E}\left(\mathrm{OR}_{\mathrm{E}}=\exp \left(\beta_{E}\right)\right)$ equal to 1 or 2 .

To model a deleterious effect size higher for rarer variants than for more common ones, we modeled the association with $\mathrm{D}\left(\beta_{G k}\right)$ and the $\mathrm{G} \times \mathrm{E}$ interaction coefficient $\left(\beta_{I k}\right)$ for each variant $k$ as a function of its population $\operatorname{MAF}\left(f_{k}\right)$, as in [12].

$$
\begin{aligned}
& \beta_{G k}=\log \left(1+\left(\mathrm{OR}_{\mathrm{G}}-1\right)\left(\frac{f_{G}}{f_{k}}\right)^{1 / 3}\right) \\
& \beta_{I k}=\log \left(1+\left(\mathrm{OR}_{\mathrm{I}}-1\right)\left(\frac{f_{G}}{f_{k}}\right)^{1 / 3}\right)
\end{aligned}
$$

where $\mathrm{OR}_{\mathrm{G}}$ and $\mathrm{OR}_{\mathrm{I}}$ are the reference odds ratios for the genetic association and $\mathrm{G} \times \mathrm{E}$ interaction of a variant with a MAF equal to $f_{G}$, respectively, and $f_{G}$ is the median of the MAF distribution of all variants with a MAF between $10^{-3}$ and $0.05\left(f_{G}=0.0012\right)$. For example, when $\mathrm{OR}_{\mathrm{G}}=2$, variants with a MAF of $3 \times 10^{-4}, 0.01$, or 0.05 were assigned an $\mathrm{OR}_{\mathrm{Gk}}$ (i.e. $\exp \left(\beta_{G k}\right)$ ) equal to $2.59,1.49$, or 1.28 , respectively. The different disease models included $\mathrm{OR}_{\mathrm{G}}$ values equal to 1 or 2 and $\mathrm{OR}_{\mathrm{I}}$ values ranging between 1 and 3 .
The power and type 1 error of each method were computed as the proportion or regions where the association was significant at $\alpha=0.05$, under alternative and null models, respectively. In scenario $1,2,100,420$, and 105 regions of 20,100 , and 400 variants were available, respectively; whereas in scenario $2,1,700$ regions of 20 rare variants (population MAF $<0.05$ ) were available. All simulations and analyses were done using $\mathrm{R}$ version 2.13.0 [31] and the package SKAT v0.77 [20, 26, 28].

\section{Results}

\section{Type 1 Error Rates}

The type 1 error of all methods is well controlled at $\alpha=0.05$ (table 2). Min-P-g has a slightly elevated type 1 error for regions of 20 variants with $\mathrm{OR}_{\mathrm{E}}=1$, whereas CAST has an elevated type 1 error of $0.074(p=0.03)$ for regions of 100 variants with $\mathrm{OR}_{\mathrm{E}}=2.0$. To further investigate the distribution of $\mathrm{p}$ values obtained with the different methods under $H_{0}$, we did quantile-quantile plots that showed no deviation from the expected 95\% confidence interval region. We also estimated the type 1 error at $\alpha=0.01$, and none of the observed type 1 errors were significantly different from the expected value (data not shown). Overall, considering the relatively small number of tests done, the quantile-quantile plots, the results at $\alpha=0.01$, and the fact that Min-P is a permutation-based test, those slight deviations are not alarming. 
Table 2. Empirical type 1 error of the different methods at $\alpha=0.05$

\begin{tabular}{|c|c|c|c|c|c|c|c|c|c|c|c|c|c|c|}
\hline Scenario & $m$ & $\mathrm{OR}_{\mathrm{E}}$ & $-g$ & -ge & $-g$ & -ge & $-g$ & -ge & $-g$ & -ge & $-g$ & -ge & $-g$ & -ge \\
\hline \multirow{3}{*}{1} & & 2.0 & 0.054 & 0.058 & 0.050 & 0.047 & 0.053 & 0.058 & 0.055 & 0.057 & 0.053 & 0.056 & 0.053 & 0.059 \\
\hline & 100 & 1.0 & 0.038 & 0.045 & 0.043 & 0.052 & 0.064 & 0.057 & 0.048 & 0.048 & 0.052 & 0.058 & 0.055 & 0.057 \\
\hline & & 2.0 & 0.038 & 0.038 & 0.067 & 0.067 & 0.048 & 0.038 & 0.057 & 0.086 & 0.029 & 0.048 & 0.057 & 0.086 \\
\hline \multirow[t]{2}{*}{2} & 20 & 1.0 & 0.043 & 0.048 & 0.047 & 0.052 & 0.051 & 0.051 & 0.052 & 0.050 & 0.046 & 0.047 & 0.049 & 0.053 \\
\hline & & 2.0 & 0.041 & 0.049 & 0.049 & 0.050 & 0.055 & 0.049 & 0.051 & 0.047 & 0.045 & 0.041 & 0.047 & 0.046 \\
\hline
\end{tabular}

Bold values are significantly different from the expected nominal value of 0.05 (bilateral $z$ test, $\alpha=0.05$ ).

In scenario 1 , the type 1 error is averaged over $2,100,420$, and 105 regions of 20,100 , and 400 variants, respectively, whereas in scenario 2, it is averaged over 1,700 regions of 20 variants.

Sequence Kernel Association Test using a weighted burden test (Burden), a weighted linear kernel (SKAT) or the optimal test (SKAT-O).
- $g$ = Test of the genetic variants only; - ge $=$ joint test of the genetic variants and the $\mathrm{G} \times \mathrm{E}$ interaction; $m=$ number of variants per region; $\mathrm{OR}_{\mathrm{E}}=$ odds ratio of disease association with the environmental factor. Fixed parameters: baseline risk of disease $\mathrm{B}=0.01$; exposure prevalence $f_{E}=0.3$.
To present power results, we first selected the configurations of CAST and SKAT that had the best overall power and compared them along with Min-P (fig. 1, 2). The comparison of the different configurations of CAST and SKAT are discussed further below and in online supplementary figures 1-4 (for all online suppl. material, see www.karger.com/doi/10.1159/000346825).

\section{Power under Scenario 1}

For a fixed proportion of causal variants (10\%), the power of all methods increases with increasing region size, genetic and environmental effects, and G×E interactions, regardless of whether a joint test of genetic association and $\mathrm{G} \times \mathrm{E}$ interaction is done or not (fig. 1). When the region contains 20 variants, the power to detect an association with the disease is very low for all methods, and accounting for $\mathrm{G} \times \mathrm{E}$ interaction neither increases nor decreases the power noticeably. When the region is larger (100 or 400 variants), the power of joint tests is about $10-20 \%$ higher than the power of the genetic association tests in situations of 'pure interaction' (i.e. $\mathrm{OR}_{\mathrm{G}}=\mathrm{OR}_{\mathrm{E}}=$ 1). In these cases, SKAT-O-ge and CAST-ge with a threshold at 0.05 and an additive coding have the highest power (fig. 1, first 3 panels at the top). In all other situations of scenario 1, the joint tests are either worse than or as good as their corresponding genetic association tests. Overall, SKAT-O-g has the best power to detect association, ex- cept for situations of 'pure interaction', where a joint test improves power (SKAT-O-ge or CAST-ge). Min-P has the lowest power, and accounting for $\mathrm{G} \times \mathrm{E}$ interaction decreased it further.

\section{Power under Scenario 2}

For small regions $(m=20)$ and increasing proportions of causal variants, the joint tests have better power than the corresponding genetic association tests (up to $20 \%$ increase) in the presence of 'pure interaction' (fig. 2). Compared to genetic association tests, the power of joint tests is similar in the presence of a main genetic or environmental effect, and slightly lower in the presence of an effect of both factors (about 2-5\% decrease). SKAT-O-g and SKAT-O-ge have the best power overall, with a slight advantage for SKAT-O-g in the presence of genetic and environmental main effects, but a larger advantage to SKAT-O-ge, when no main genetic or environmental effects are present. Here again, Min-P has much lower power and the joint test improved the power only minimally in the presence of 'pure interaction'.

In both scenarios, when no $\mathrm{G} \times \mathrm{E}$ interaction is present but the genetic variants have an effect $\left(\mathrm{OR}_{\mathrm{G}}=2\right.$ and $\mathrm{OR}_{\mathrm{I}}=1$ ), the power of joint tests is $5-10 \%$ smaller than the power of genetic association tests, regardless of the method used. 


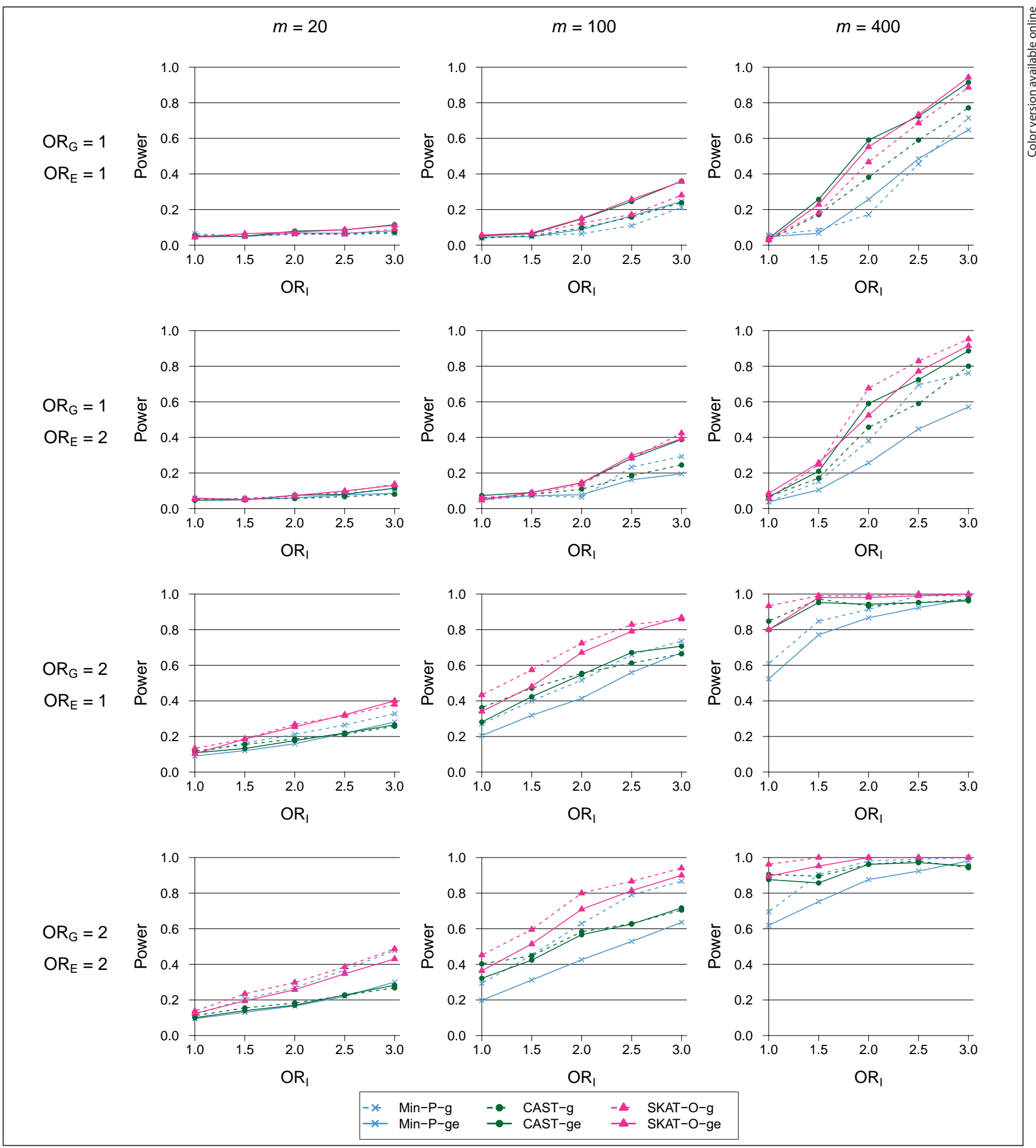

Fig. 1. Power to detect association with the genetic region as a function of the $\mathrm{G} \times \mathrm{E}$ interaction $\left(\mathrm{OR}_{\mathrm{I}}\right)$ under scenario 1 at $\alpha=0.05$. CAST: used with an additive coding of the score $S_{i}$ and with a threshold $\mathrm{T}=0.05$. SKAT-O: used with the option 'optimal' and Beta(MAF, 1, 25) weights. The panels are arranged in columns by region size $(m)$ and in rows by disease model, genetic effect $\left(\mathrm{OR}_{\mathrm{G}}\right)$ and environmental effect $\left(\mathrm{OR}_{\mathrm{E}}\right)$. Fixed parameters: proportion of causal variants $=10 \%$; baseline risk of disease $\mathrm{B}=0.01$; exposure prevalence $f_{E}=0.3$. 


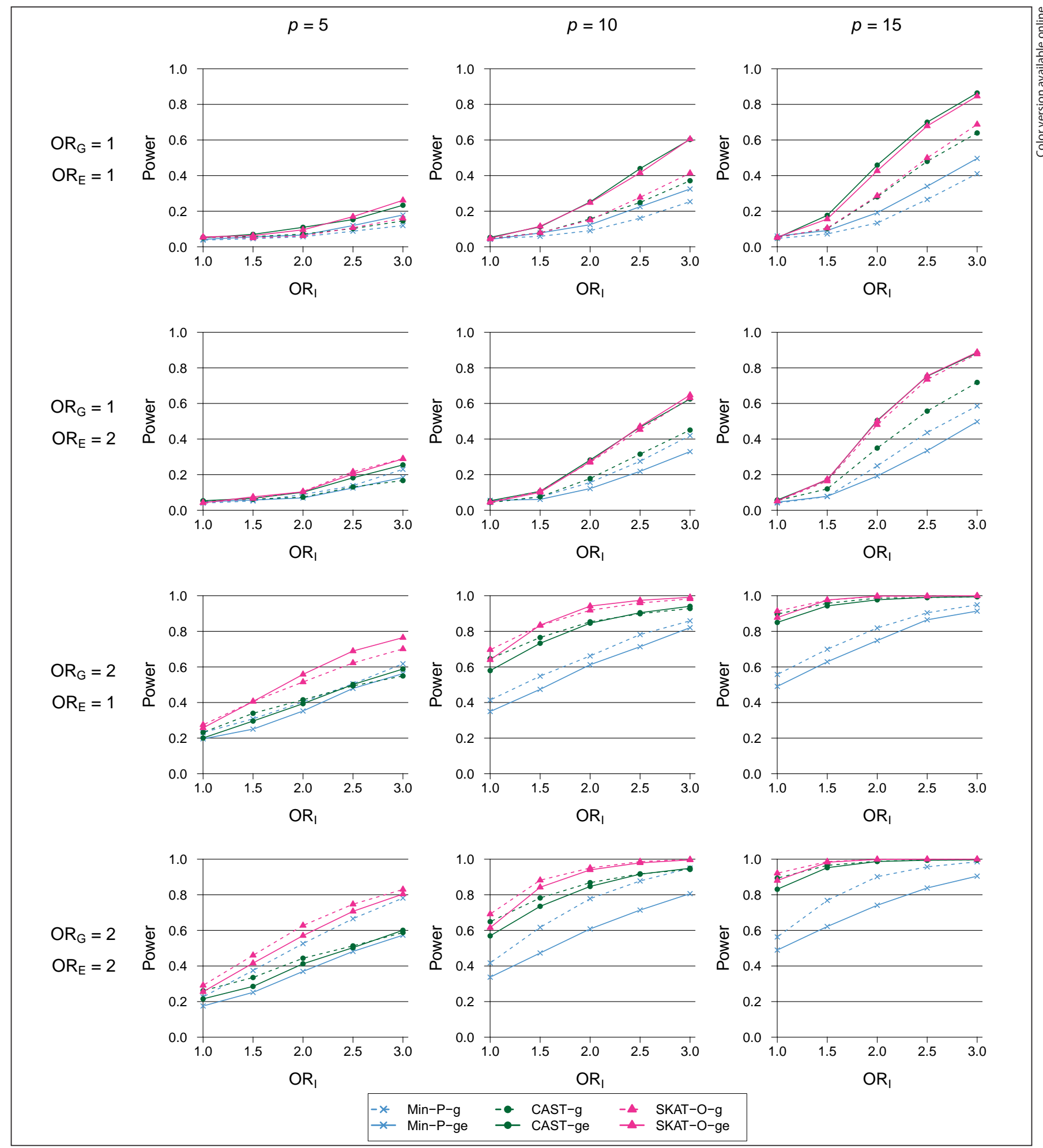

Fig. 2. Power to detect association with the genetic region as a function of the $\mathrm{G} \times \mathrm{E}$ interaction $\left(\mathrm{OR}_{\mathrm{I}}\right)$ under scenario 2 at $\alpha=0.05$. CAST: used with an additive coding of the score $S_{i}$ and with a threshold $\mathrm{T}=0.05$. SKAT-O: used with the option 'optimal' and Beta(MAF, 1, 25) weights. The panels are arranged in columns by causal variant count $(p)$ and in rows by disease model, genetic effect $\left(\mathrm{OR}_{\mathrm{G}}\right)$ and environmental effect $\left(\mathrm{OR}_{\mathrm{E}}\right)$. Fixed parameters: region size $m=20$ variants; baseline risk of disease $\mathrm{B}=0.01$; exposure prevalence $f_{E}=0.3$.
Gene-Environment Interactions and Rare Variants 


\section{Configurations of CAST}

Among the different configurations used for CAST (combinations of threshold and coding for $S_{i}$ ), the choice of the threshold had a noteworthy impact on the power, regardless of the coding used. When the threshold underestimates the range of population MAF of causal variants $(\mathrm{T}=0.01)$, the power of CAST decreases importantly (online suppl. fig. 1,2). Overall, the tests using a threshold $\mathrm{T}=0.05$ had better power than when $\mathrm{T}=0.01$. The difference in power between tests based on a binary or an additive coding was much less important. The additive coding had just a slight advantage over the binary coding, in the absence of $\mathrm{G} \times \mathrm{E}$ interaction and in the presence of main genetic or environmental effects.

\section{Configurations of SKAT}

Interestingly, the aim of SKAT-O, to unify the advantages of Burden and SKAT, has clearly been achieved. Indeed, the power of SKAT-O-g and SKAT-O-ge are uniformly equal or superior to the power of the corresponding tests in Burden and SKAT. In scenario 1, SKAT had higher power than Burden, whereas in scenario 2, Burden had a higher power than SKAT (online suppl. fig. 3, 4).

\section{Discussion}

\section{When Does Accounting for $G \times E$ Interaction Help?}

Accounting for $\mathrm{G} \times \mathrm{E}$ interactions when testing for disease association with grouped rare variants can increase power when the interacting factors do not have independent main effects (scenario $1, \mathrm{OR}_{\mathrm{G}}=\mathrm{OR}_{\mathrm{E}}=1$ ), or when the proportion of causal variants is high (scenario 2). In the presence of a main genetic or environmental effect and when the proportion of causal variants is small, accounting for $\mathrm{G} \times \mathrm{E}$ interaction does not increase power, and sometimes decreases it. One explanation is that even for a strong $\mathrm{G} \times \mathrm{E}$ interaction, only a very small subset of subjects will both carry the rare allele and be exposed, and so the interaction will have limited impact in comparison with the main genetic or environmental effect. Although pure $\mathrm{G} \times \mathrm{E}$ interactions are plausible [32-34], their role and impact are probably limited in the realm of possible disease models. Consequently, this approach does not seem very promising for the primary analysis of the association of rare genetic variants with a disease. In terms of power, it is not worthwhile to incorporate $\mathrm{G} \times \mathrm{E}$ interactions into the analysis of rare variants when no interaction exists.
However, when variants are selected based on specific criteria that might increase the proportion of causal variants, the joint test can be a good choice. Using for example SKAT-O-ge, the decrease in power in the absence of $\mathrm{G} \times \mathrm{E}$ interactions is limited, while the power increase in the presence of $\mathrm{G} \times \mathrm{E}$ interactions is important.

Overall, these results agree with findings for common variants $[22,23]$, but the impact of the low frequency of the genetic variants makes the joint test less attractive than for common variant discovery. We postulate that if the prevalence of the environmental factor is lower than in our simulations $\left(f_{E}=0.3\right)$, the power of the joint test relative to the genetic association test will be even lower.

\section{Which Method to Use?}

We have extended and compared three new methods to jointly test genetic association and $\mathrm{G} \times \mathrm{E}$ interaction for rare variants. Min-P had the lowest power in our simulated models and had the longest computational time, so it may be inappropriate for large datasets. The power of Min-P relative to the other methods is more sensitive to variations in the proportion of causal variants, a factor rarely known in practice. Moreover, the version of Min-P implemented here does not allow using continuous outcomes or adjusting for covariates. Although it is much faster, can adjust for covariates, and can be applied for continuous exposures and quantitative traits, the power of CAST, whether using a binary or additive coding, was sensitive to the arbitrary choice of the threshold below which variants are aggregated. This issue has been addressed in the literature by using weighting schemes or variable threshold approaches $[15,19]$, though we did not incorporate extensions of these methods and others in our comparison. Finally, the weighted SKAT-O had the best overall power and a short computational time. SKAT$\mathrm{O}$ can adjust for covariates and analyze continuous exposures and quantitative traits. This unified approach solves the issue of the kernel selection by combining the linear model with a burden-based model. Nonetheless, the weighting choice introduces implicit assumptions that could impact power.

For $\alpha=0.01$, all powers decrease but the relative power of the different methods are similar (data not shown). Due to the computational burden in particular for regions of large size, we had to limit the number of regions studied and thus could not assess the power at genomewide levels, which would be important to evaluate. However, based on a previous comparison between SKAT and Burden tests, we expect that the relative power of the different methods will be similar [20]. 


\section{Potential Issues}

As in any simulation study, our analysis made several assumptions that may be violated in practice. First, we assumed that genetic and $\mathrm{G} \times \mathrm{E}$ interaction effects were in the same direction. Although the common disease - rare variants hypothesis suggests that rare variants most probably are deleterious since they are selected against, rare variants with opposite main effects, opposite $G \times E$ interactions effects, or $\mathrm{G} \times \mathrm{E}$ interactions opposite to the genetic main effects cannot be ruled out. By making no assumption on the direction of effects, SKAT would be better adapted than burden tests to handle genetic and $\mathrm{G} \times \mathrm{E}$ interaction effects with heterogeneous directions.

Second, when modeling the $\mathrm{G} \times \mathrm{E}$ interaction, we assumed that all associated variants had the same magnitude of interactions with E. If only a subset of the associated variants interacts with $\mathrm{E}$, the power of joint tests is expected to be lower than the power obtained in our models.

Third, for the three tests (Burden-ge, SKAT-ge, and SKAT-O-ge), we used a single kernel for both the genetic and the $\mathrm{G} \times \mathrm{E}$ interaction components. These three tests are score tests from random genetic and $\mathrm{G} \times \mathrm{E}$ interaction effects models. Their likelihoods are identical to the likelihoods of the three corresponding tests of genetic effects only (Burden-g, SKAT-g, and SKAT-O-g), except that the number of elements in the genotype matrix is doubled by adding for each variant $\mathrm{G} \times \mathrm{E}$ interaction scores, i.e. for each individual the product of his genotype at this variant and his exposure status. The weights for the half matrix containing the $\mathrm{G} \times \mathrm{E}$ interactions are computed using the distribution $\operatorname{Beta}\left(f_{\mathrm{G} \times \mathrm{E}}, a_{1}=1, a_{2}=25\right)$, where $f_{\mathrm{G} \times \mathrm{E}}$ is the relative frequency of the $\mathrm{G} \times \mathrm{E}$ interaction terms. A consequence of this ad-hoc choice is that weights of $\mathrm{G} \times \mathrm{E}$ interactions are higher than those of the genetic effects. Since SKAT allows all element of the matrix to have independent effects, our joint test of genetic association and $\mathrm{G} \times \mathrm{E}$ interaction is valid. However, it would be valuable to model the genetic effect and the GXE interaction separately and to evaluate the use of different weighting schemes for the two components and testing for $\mathrm{G} \times \mathrm{E}$ interaction only.

Fourth, our models only explored a baseline risk of disease set to 0.01 . However, previous comparisons between SKAT and Burden tests have shown that the lower the disease prevalence, the higher the power. Indeed, the case-control design enriches samples with causal variants when the baseline risk of disease is low more than when it is high. However, this variation in power is minimal compared to the difference of power between methods [20].

Finally, this simulated allele frequency spectrum is skewed slightly higher than those observed in recent large-scale sequencing studies [6, 7]. A possible explanation is that the population genetic model used to simulate these data did not account for the rapid population growth suggested to be responsible for the excess of rare variants [5]. This may decrease the power of all methods, but this decrease would be more important for the Min-P tests, which rely on the presence of at least one strong effect, than for the CAST or SKAT tests.

In conclusion, when testing for disease association with a set of rare variants, accounting for $\mathrm{G} \times \mathrm{E}$ interactions can improve power in specific situations (pure interaction or high proportion of causal variants interacting with the environment). However, this approach is not appropriate for a primary analysis. Among the methods compared, the optimized and weighted SKAT performed best whether to test for genetic association or to test it jointly with $\mathrm{G} \times \mathrm{E}$ interactions.

\section{Acknowledgements}

This work was funded by National Institutes of Health grants (R01 CA88164, U01 CA127298, and R25 CA112355).

\section{References}

$>1$ Eichler EE, Flint J, Gibson G, Kong A, Leal SM, Moore JH, Nadeau JH: Missing heritability and strategies for finding the underlying causes of complex disease. Nat Rev Genet 2010;11:446-450

$\checkmark 2$ Manolio TA, Collins FS, Cox NJ, et al: Finding the missing heritability of complex diseases. Nature 2009;461:747-753.

$\checkmark 3$ Mechanic LE, Chen HS, Amos CI, et al: Next generation analytic tools for large scale genet-

Gene-Environment Interactions and Rare Variants ic epidemiology studies of complex diseases. Genet Epidemiol 2012;36:22-35.

4 van Ijzendoorn MH, Bakermans-Kranenburg MJ, Belsky J, Beach S, Brody G, Dodge KA, Greenberg M, Posner M, Scott S: Gene-byenvironment experiments: a new approach to finding the missing heritability. Nat Rev Genet $2011 ; 12: 881$; author reply 881 .

5 Keinan A, Clark AG: Recent explosive human population growth has resulted in an excess of rare genetic variants. Science 2012;336:740743.

6 Nelson MR, Wegmann D, Ehm MG, et al: An abundance of rare functional variants in 202 drug target genes sequenced in 14,002 people. Science 2012;337:100-104

7 Tennessen JA, Bigham AW, O'Connor TD, et al: Evolution and functional impact of rare coding variation from deep sequencing of human exomes. Science 2012;337:64-69. 
8 Gibson G: Rare and common variants: twenty arguments. Nat Rev Genet 2011;13:135-145.

-9 Basu S, Pan W: Comparison of statistical tests for disease association with rare variants. Genet Epidemiol 2011;35:606-619.

$>10$ Stitziel NO, Kiezun A, Sunyaev S: Computational and statistical approaches to analyzing variants identified by exome sequencing. Genome Biol 2011;12:227.

-11 Ladouceur M, Dastani Z, Aulchenko YS, Greenwood CM, Richards JB: The empirical power of rare variant association methods: results from sanger sequencing in 1,998 individuals. PLoS Genet 2012;8:e1002496.

12 Hoffmann TJ, Marini NJ, Witte JS: Comprehensive approach to analyzing rare genetic variants. PLoS One 2010;5:e13584.

-13 Kazma R, Hoffmann TJ, Witte JS: Use of principal components to aggregate rare variants in case-control and family-based association studies in the presence of multiple covariates. BMC Proc 2011;5(suppl 9):S29.

14 Li B, Leal SM: Methods for detecting associations with rare variants for common diseases: application to analysis of sequence data. Am J Hum Genet 2008;83:311-321.

15 Madsen BE, Browning SR: A groupwise association test for rare mutations using a weighted sum statistic. PLoS Genet 2009;5:e1000384.

16 Morgenthaler S, Thilly WG: A strategy to discover genes that carry multi-allelic or monoallelic risk for common diseases: a cohort allelic sums test (CAST). Mutat Res 2007;615: 28-56.
17 Morris AP, Zeggini E: An evaluation of statistical approaches to rare variant analysis in genetic association studies. Genet Epidemiol 2010;34:188-193.

18 Neale BM, Rivas MA, Voight BF, Altshuler D, Devlin B, Orho-Melander M, Kathiresan S, Purcell SM, Roeder K, Daly MJ: Testing for an unusual distribution of rare variants. PLoS Genet 2011;7:e1001322.

19 Price AL, Kryukov GV, de Bakker PI, Purcell SM, Staples J, Wei LJ, Sunyaev SR: Pooled association tests for rare variants in exon-resequencing studies. Am J Hum Genet 2010;86: 832-838.

20 Wu MC, Lee S, Cai T, Li Y, Boehnke M, Lin $\mathrm{X}$ : Rare-variant association testing for se quencing data with the sequence kernel association test. Am J Hum Genet 2011;89:82-93.

21 Asimit J, Zeggini E: Rare variant association analysis methods for complex traits. Annu Rev Genet 2010;44:293-308.

22 Kazma R, Babron MC, Genin E: Genetic association and gene-environment interaction: a new method for overcoming the lack of exposure information in controls. Am J Epidemiol 2011;173:225-235.

-23 Kraft P, Yen YC, Stram DO, Morrison J, Gauderman WJ: Exploiting gene-environment interaction to detect genetic associations. Hum Hered 2007;63:111-119.

24 Westfall P, Young S: On adjusting P-values for multiplicity. Biometrics 1993;49:941-945

25 Fisher R: On the interpretation of $\chi^{2}$ from contingency tables, and the calculation of P. J Royal Stat Soc 1922;85:87-94.
$26 \mathrm{Wu}$ MC, Kraft P, Epstein MP, Taylor DM, Chanock SJ, Hunter DJ, Lin X: Powerful SNPset analysis for case-control genome-wide association studies. Am J Hum Genet 2010;86: 929-942.

27 Davies R: The distribution of a linear combination of chi-square random variables. J $\mathrm{R}$ Stat Soc Ser C Appl Stat 1980;29:323-333.

28 Lee S, Wu MC, Lin X: Optimal tests for rare variant effects in sequencing association studies. Biostatistics 2012;13:762-775.

29 Cardin NJ, Mefford JA, Witte JS: Joint association testing of common and rare genetic variants using hierarchical modeling. Genet Epidemiol 2012;36:642-651.

30 Hernandez RD: A flexible forward simulator for populations subject to selection and demography. Bioinformatics 2008;24:27862787.

31 R Development Core Team: R: a language and environment for statistical computing. Vienna, Austria, 2005. http://www.R-project.org.

32 Hahn LW, Ritchie MD, Moore JH: Multifactor dimensionality reduction software for detecting gene-gene and gene-environment interactions. Bioinformatics 2003;19:376-382.

33 Manuguerra M, Matullo G, Veglia F, et al: Multi-factor dimensionality reduction applied to a large prospective investigation on gene-gene and gene-environment interactions. Carcinogenesis 2007;28:414-422.

-34 Ottman R: Gene-environment interaction: definitions and study designs. Prev Med 1996; 25:764-770. 